\title{
Heavy hadrons spectra on lattice using NRQCD
}

\author{
Protick Mohanta $^{1, \star}$ and Subhasish Basak ${ }^{1}$ \\ ${ }^{1}$ National Institute of Science Education and Research Bhubaneswar, P.O. Jatni, Khurda 752050, Odisha, \\ India
}

\begin{abstract}
Comparison of radial excitation energies to masses show that the velocity of $b$ quark is very non-relativistic in bottomonium states. In a mixed system like charmed B meson, the b quark has less velocity than it has in bottomonium states and in strange $\mathrm{B}$ meson it is even slower. So one can use NRQCD for the b quark in those systems. Using overlap and hisq action for the $\mathrm{s}$ and $\mathrm{c}$ quarks and NRQCD for $\mathrm{b}$ quark we simulated spectra of charmed and strange B mesons and also few other baryons.
\end{abstract}

\section{Introduction}

Lattice methods are powerful techniques in analyzing the spectrum of hadrons. However for hadrons containing heavy quarks particularly bottom quark are difficult to analyze. For spectrum calculation it is necessary that $a M<<1$. For light quarks it is true but for charm quark $a M_{c}>0.7$ and for bottom quark $a M_{b}>2$ with lattice spacing $a=0.12 \mathrm{fm}$. However in hadrons containing heavy quarks the velocities of heavy quarks are non-relativistic. Comparison of masses of heavy quarkonium to the masses of heavy quarks also indicates the same. For example $M_{\Upsilon}=9390 \mathrm{MeV}$ whereas $2 \times M_{b}=8360 \mathrm{MeV}(\overline{M S}$ Scheme $)$ and $M_{J / \psi}=3096 \mathrm{MeV}$ whereas $2 \times M_{c}=2580$ $\mathrm{MeV}$. This is in contrast with mesons and baryons that are made up of light quarks for example mass of pions $M_{\pi} \sim 140 \mathrm{MeV}$ whereas masses of up or down quark is few MeV. So one can use effective theories like non relativistic QCD(NRQCD) to study hadrons containing heavy quark/quarks.

In this report we present some of preliminary results on charmed and strange B meson and baryons. For the bottom quark we used NRQCD action corrected up to order $O\left(v^{6}\right)$, for strange and charm quarks we used overlap and hisq actions. We simulated the ground state of $B_{c}$ and $B_{s}$ meson, hyperfine splitting of $\Upsilon$ and $\eta_{b}$. We also simulated ground states of $\Omega_{b b b}\left(\frac{3}{2}^{+}\right), \Omega_{b b s}^{*}\left(\frac{3}{2}^{+}\right)$and $\Lambda_{b s s}\left(\frac{1}{2}^{+}\right)$baryons.

\section{Simulation Details}

\subsection{NRQCD Action}

We used NRQCD for the bottom quark. The NRQCD Lagrangian [1], [2]corrected up to $O\left[(v / c)^{6}\right.$ ] is given by $\mathcal{L}=\mathcal{L}_{0}+\delta \mathcal{L}_{v^{4}}+\delta \mathcal{L}_{v^{6}}$ where

$$
\mathcal{L}_{0}=\psi(x)^{\dagger}\left(i D_{0}+\frac{\vec{D}^{2}}{2 m}\right) \psi(x)
$$

${ }^{\star}$ Speaker, e-mail: protick.m@niser.ac.in 


$$
\begin{aligned}
\delta \mathcal{L}_{v^{4}}= & c_{1} \frac{1}{8 m^{3}} \psi^{\dagger} D^{4} \psi+c_{2} \frac{g}{8 m^{2}} \psi^{\dagger}(\vec{D} \cdot \vec{E}-\vec{E} \cdot \vec{D}) \psi \\
& +c_{3} \frac{i e}{8 m^{2}} \psi^{\dagger} \vec{\sigma} \cdot(\vec{D} \times \vec{E}-\vec{E} \times \vec{D}) \psi+c_{4} \frac{g}{2 m} \psi^{\dagger} \vec{\sigma} \cdot \vec{B} \psi \\
\delta \mathcal{L}_{v^{6}}= & c_{5} \frac{g}{8 m^{3}} \psi^{\dagger}\left\{\vec{D}^{2}, \vec{\sigma} \cdot \vec{B}\right\} \psi-c_{6} \frac{i g^{2}}{8 m^{3}} \psi^{\dagger}(\vec{\sigma} \cdot \vec{E} \times \vec{E}) \psi \\
& +c_{7} \frac{3}{64} \frac{i g}{m^{4}} \psi^{\dagger}\left\{\vec{D}^{2}, \vec{\sigma} \cdot(\vec{D} \times \vec{E}-\vec{E} \times \vec{D})\right\} \psi
\end{aligned}
$$

Tree level value of all these coefficients $c_{1}, c_{2}, c_{3}, c_{4}, c_{5}, c_{6}$ and $c_{7}$ is 1 . Here $D$ denotes the continuum covariant derivative. The lattice version of NRQCD is obtained by replacing the continuum derivatives by lattice derivatives:

$$
a \Delta_{\mu}^{+} \psi(x)=U_{\mu}(x) \psi(x+a \hat{\mu})-\psi(x) ; a \Delta_{\mu}^{-} \psi(x)=\psi(x)-U_{\mu}^{\dagger}(x-a \hat{\mu}) \psi(x-a \hat{\mu})
$$

Symmetric derivative and Laplacian is related to forward and backward derivative as

$$
\Delta^{ \pm}=\frac{1}{2}\left(\Delta^{+}+\Delta^{-}\right) ; \Delta^{2} \equiv \sum_{i} \Delta_{i}^{+} \Delta_{i}^{-}=\sum_{i} \Delta_{i}^{-} \Delta_{i}^{+}
$$

Now for coarser lattices in order to extract physical quantities we need to improve the discretization error. Simply by Taylor expansion of symmetric derivative and the Laplacian operator we can find their forms corrected up to $O\left(a^{4}\right)$ [1]

$$
\tilde{\Delta}_{i}^{ \pm} f=\Delta_{i}^{ \pm} f-\frac{a^{2}}{6} \Delta_{i}^{+} \Delta_{i}^{ \pm} \Delta_{i}^{-} f ; \tilde{\Delta}^{2}=\Delta^{2}-\frac{a^{2}}{12} \sum_{i}\left[\Delta_{i}^{+} \Delta_{i}^{-}\right]^{2}
$$

Similarly using cloverleaf instead of plaquette one can improve the gauge fields up to $O\left(a^{4}\right)$

$$
g \tilde{F}_{\mu \nu}(x)=g F_{\mu \nu}(x)-\frac{a^{4}}{6}\left[\Delta_{\mu}^{+} \Delta_{\mu}^{-}+\Delta_{v}^{+} \Delta_{v}^{-}\right] g F_{\mu \nu}(x)
$$

NRQCD propagators of the quarks are obtained by the time evolution of the Green's function

$$
G(\vec{x}, t+1 ; \overrightarrow{0}, 0)=\left(1-\frac{a H_{0}}{2 n}\right)^{n}\left(1-\frac{a \delta H}{2}\right) U_{t}(x, t)^{\dagger}\left(1-\frac{a \delta H}{2}\right)\left(1-\frac{a H_{0}}{2 n}\right)^{n} G(\vec{x}, t ; \overrightarrow{0}, 0)
$$

with $G(\vec{x}, t ; \overrightarrow{0}, 0)=0$ for $t<0$ and $G(\vec{x}, t ; \overrightarrow{0}, 0)=\delta_{\vec{x}, 0}$ for $t=0$. From the above equation it is evident that $n>\frac{3}{2 m}$. In NRQCD quarks and anti-quarks are decoupled. Here $G(\vec{x}, t ; \overrightarrow{0}, 0)$ is a $2 \times 2$ matrix in spinor space. The propagators of heavy anti-quarks can be obtained by taking the dagger of the quark propagators [2].

\subsection{Correlators}

When using NRQCD, all energies obtained from fits to hadronic two-point functions are shifted by some common constant, as the rest mass is not included in the Lagrangian eq(1),eq(2) and eq(3). Thus, to tune the b quark mass we have to use the 'kinetic mass'

$$
a M_{k i n}=\frac{(a p)^{2}-(a \Delta E)^{2}}{2 a \Delta E}
$$


In order to tune kinetic mass given in eq(5) we need to calculate the energy of the particle for a given momentum. Correlator for $\Upsilon$ or $\eta_{b}$ is $[3,4]$

$$
C(\vec{p}, t)=\sum_{\vec{x}} e^{i \vec{p} \cdot \vec{x}} \operatorname{Tr}\left[\mathrm{G}^{\dagger}(\mathrm{x}, 0) \Gamma_{\mathrm{sk}}(\mathrm{x}) \mathrm{G}(\mathrm{x}, 0) \Gamma_{\mathrm{sc}}^{\dagger}(0)\right]
$$

Here $\Gamma(x)=\Omega \phi(x)$. $\phi$ is the smearing operator and $\Omega$ is a $2 \times 2$ matrix in spin space. $\Omega=I$ for pseudoscalar particles and $\Omega=\sigma_{i}$ for vector particles [5].

Correlator for heavy-light mesons for example $B_{s}$ or $B_{c}$ meson is given as [6]

$$
C(\vec{p}, t)=-\sum_{\vec{x}} e^{i \vec{p} \cdot \vec{x}} \operatorname{Tr}\left[\gamma_{5} \mathrm{M}(\mathrm{x}, 0)^{\dagger} \gamma_{5} \Gamma \mathrm{G}(\mathrm{x}, 0) \Gamma\right]
$$

$\Gamma=\gamma_{5}$ for pseudoscalar and $\Gamma=\gamma_{i}$ for vector particles. $M(x, 0)$ is the propagator of the relativistic charm or strange quark. $G(x, 0)$ is now a $4 \times 4$ matrix in spinor space having vanishing lower components but it is in Dirac representation of gamma matrices. We can convert it to MILC gamma representation by a unitary transformation

$$
S=\frac{1}{\sqrt{2}}\left(\begin{array}{cc}
\sigma_{y} & \sigma_{y} \\
-\sigma_{y} & \sigma_{y}
\end{array}\right)
$$

For $\Omega_{b b b}\left(\frac{3}{2}^{+}\right)$the interpolator is $\left(O_{k}\right)_{\alpha}=\epsilon_{a b c}\left(Q^{a T} C \gamma_{k} Q^{b}\right) Q_{\alpha}^{c}$ with $C=\gamma_{4} \gamma_{2}$, and the correlator is given by [7]

$$
\begin{aligned}
C_{i j, \alpha \beta}(t) & =\sum_{\vec{x}}\left\langle 0\left|\left[O_{i}(\vec{x}, t)\right]_{\alpha}\left[O_{j}^{\dagger}(\overrightarrow{0}, 0)\right]_{\beta}\right| 0\right\rangle \\
& =\sum_{\vec{x}} \epsilon_{a b c} \epsilon_{f g h} G_{\alpha \beta}^{c h}(x, 0) \operatorname{Tr}\left[\mathrm{C} \gamma_{\mathrm{i}} \mathrm{G}^{\mathrm{bg}}(\mathrm{x}, 0) \overline{\mathrm{C} \gamma_{\mathrm{j}}} \mathrm{G}^{\mathrm{af}}(\mathrm{x}, 0)\right]
\end{aligned}
$$

Here $i, j$ are the indices of the gamma matrices $\left(\gamma_{i}, \gamma_{j}\right)$ and $\alpha, \beta$ are the spinor indices. In the last line of the above equation we haven taken transpose only over spin indices. The correlator has overlap with both spin $3 / 2$ and spin $1 / 2$ states: $C_{i j}(t)=Z_{3 / 2} e^{-E_{3 / 2} t} \Pi P_{i j}^{3 / 2}+Z_{1 / 2} e^{-E_{1 / 2} t} \Pi P_{i j}^{1 / 2}$, where $\Pi=\frac{1}{2}\left(1+\gamma_{4}\right)$, $P_{i j}^{3 / 2}=\delta_{i j}-\frac{1}{3} \gamma_{i} \gamma_{j}, P_{i j}^{1 / 2}=\frac{1}{3} \gamma_{i} \gamma_{j}$ and $P_{i j}^{3 / 2} \cdot P_{j k}^{1 / 2}=0$. We can decouple their contributions by using:

$$
P_{x x}^{3 / 2} \cdot C_{x x}+P_{x y}^{3 / 2} \cdot C_{y x}+P_{x z}^{3 / 2} \cdot C_{z x}=\frac{2}{3} Z_{3 / 2} \Pi e^{-E_{3 / 2} t}
$$

For $\Omega_{b b s}^{*}\left(\frac{3}{2}^{+}\right)$the interpolator is $\left(O_{k}\right)_{\alpha}=\epsilon_{a b c}\left(Q^{a T} C \gamma_{k} Q^{b}\right) s_{\alpha}^{c}$ and the correlator is given by [8]

$$
\begin{aligned}
C_{i j, \alpha \beta}(t) & =\sum_{\vec{x}}\left\langle 0\left|\left[O_{i}(\vec{x}, t)\right]_{\alpha}\left[O_{j}^{\dagger}(\overrightarrow{0}, 0)\right]_{\beta}\right| 0\right\rangle \\
& =\sum_{\vec{x}} \epsilon_{a b c} \epsilon_{f g h}\left[M^{c h}(x, 0) \cdot \gamma_{4}\right]_{\alpha \beta} \operatorname{Tr}\left[\mathrm{C} \gamma_{\mathrm{i}} \mathrm{G}^{\mathrm{bg}}(\mathrm{x}, 0) \overline{\mathrm{C} \gamma_{\mathrm{j}}} \mathrm{G}^{\mathrm{af}}{ }^{\mathrm{T}}(\mathrm{x}, 0)\right]
\end{aligned}
$$

This correlator also has overlap with both spin $3 / 2$ and spin $1 / 2$ states and can be decoupled in the similar way.

Finally we write the interpolator of $\Lambda_{b s s}\left(\frac{1}{2}^{+}\right)$baryon, which is $O_{\alpha}=\epsilon_{a b c}\left(s^{a T} C \gamma_{5} s^{b}\right) Q_{\alpha}^{c}$ and the correlator is given by [8]

$$
\begin{aligned}
C_{\alpha \beta}(t) & =\sum_{\vec{x}}\left\langle 0\left|[O(\vec{x}, t)]_{\alpha}\left[O^{\dagger}(\overrightarrow{0}, 0)\right]_{\beta}\right| 0\right\rangle \\
& =\sum_{\vec{x}} \epsilon_{a b c} \epsilon_{f g h}\left[G^{c h}(x, 0)\right]_{\alpha \beta} \operatorname{Tr}\left[\gamma_{4} \gamma_{2} \gamma_{5} \mathrm{M}^{\mathrm{bg}}(\mathrm{x}, 0) \gamma_{5} \gamma_{2} \gamma_{4} \mathrm{M}^{\mathrm{af}}(\mathrm{x}, 0)\right]
\end{aligned}
$$

This correlator does not have any overlap with spin $3 / 2$ state. 


\section{Results}

We used a ensemble of $40,24^{3} \times 64$ HISQ lattices with $N_{f}=2+1+1$ having $m_{l} / m_{s}=1 / 5$, $a m_{c}=0.635$ and gauge coupling $10 / g^{2}=6.00$ generated by MILC collaboration. The lattice spacing is $a=0.12 \mathrm{fm}$. Details of these lattices can be found here [9]. We set all the coefficients of the NRQCD action eq(2),eq(3) $c_{1}, c_{2}, c_{3}, c_{4}, c_{5}, c_{6}$ and $c_{7}$ to be 1 and nr-loop parameter of eq(4) $n=3$. The mass parameter $m$ of the Lagrangian eq(1),eq(2), eq(3) is tuned to produce the kinetic mass of $\eta_{b}$ $=9.42 \mathrm{GeV}$. We find that the mass differences of $\Upsilon$ and $\eta_{b}$ to be $131 \mathrm{MeV}$.

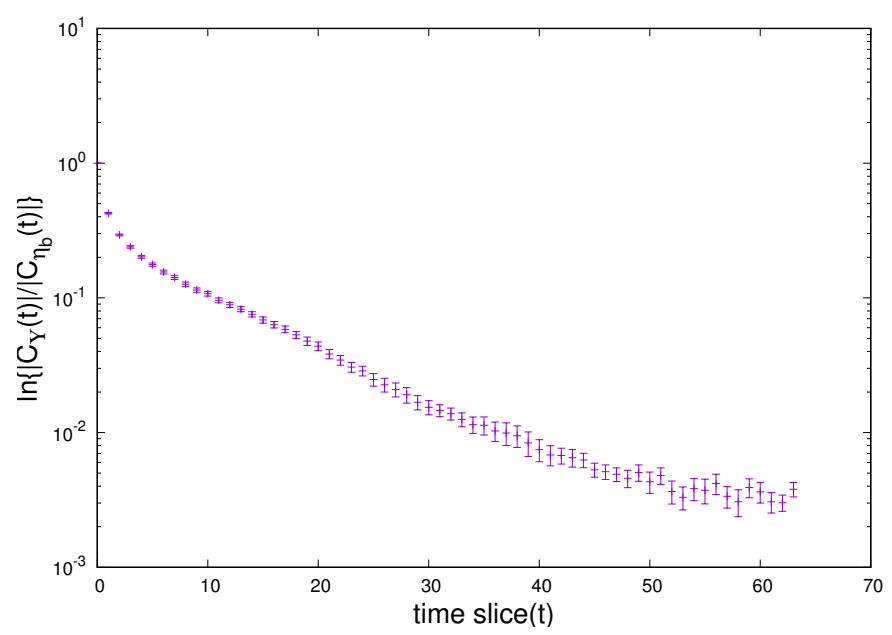

Figure 1. Hyperfine splitting of bottomonium 1S states.

We used overlap action to simulated $B_{c}$ and $B_{s}$ mesons and we used hisq action for $B_{s}$ meson only. Simulations of $B_{c}$ using hisq action is under way.

\subsection{Simulations with overlap fermions}

In overlap formalism charm quark mass has been tuned to produce kinetic mass of the $\eta_{c}$ to be $3.05 \mathrm{GeV}$. Strange quark has been directly tuned to produce $s \bar{s}$ pseudoscalar pole mass to be 686 $\mathrm{MeV}$. Here we used mass formula predicted by chiral perturbation theory to calculate the mass of pseudoscalar s-quarkonium state $M_{s \bar{s}}=\sqrt{2 M_{K}^{2}-M_{\pi}^{2}}$. This part of the tuning of strange and charm quark mass using overlap action has been done by the TIFR, Mumbai group [10]. As we used kinetic mass in tuning the bottom and charm masses the following formula has been used to calculate the mass of $B_{c}$.

$$
M_{B_{c}}=E_{B_{c}}+\frac{1}{2}\left(M_{\eta_{b}}-E_{\eta_{b}}\right)+\frac{1}{2}\left(M_{\eta_{c}}-E_{\eta_{c}}\right)
$$

Here $E_{B_{c}}, E_{\eta_{b}}, E_{\eta_{c}}$ are the simulated masses and $M_{\eta_{b}}, M_{\eta_{c}}$ are their pdg values.

We find $M_{B_{c}}=6256.5 \mathrm{Mev}$ with error $= \pm 20 \mathrm{MeV}$.

For $B_{s}$ we used the following formula

$$
M_{B_{s}}=E_{B_{s}}+\frac{1}{2}\left(M_{\eta_{b}}-E_{\eta_{b}}\right)
$$

and we found $M_{B_{s}}=5096 \mathrm{MeV} \pm 30 \mathrm{MeV}$. 


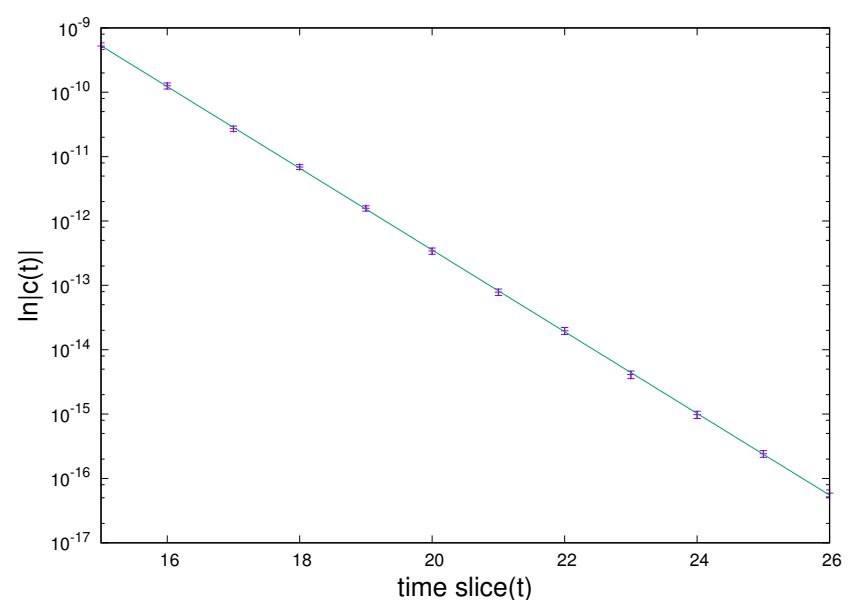

Figure 2. $B_{c}$ meson correlators obtained at zero momentum using eq(7).

\subsection{Simulations with hisq fermions}

Using hisq action we find the mass of $B_{s}$ meson to be $M_{B_{s}}=5040 \mathrm{MeV}$. For baryons we used only the hisq action for strange quark/quarks.

For $\Omega_{b b b}\left(\frac{3}{2}^{+}\right)$we used the following formula to calculate its mass

$$
M_{\Omega_{b b b}}=E_{\Omega_{b b b}}+\frac{3}{2}\left(M_{\eta_{b}}-E_{\eta_{b}}\right)
$$

We find $M_{\Omega_{b b b}}=14.38 \mathrm{GeV}$ with error $= \pm 20 \mathrm{MeV}$.

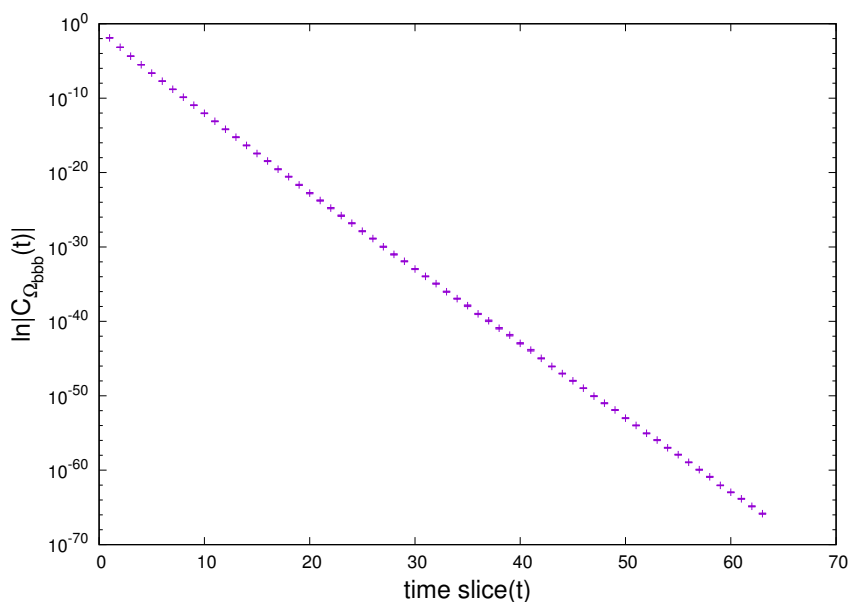

Figure 3. $\Omega_{b b b}$ baryon correlators obtained by using eq(9). Spin indices has been summed over: $C_{\Omega_{b b b}}(t)=$ $\sum_{\alpha \beta}\left[P_{x k}^{3 / 2} \cdot C_{k x}\right]_{\alpha \beta}$. Here we assumed the summation convention for repeated indices.

For $\Omega_{b b s}^{*}\left(\frac{3}{2}^{+}\right)$we find $M_{\Omega_{b b s}}=E_{\Omega_{b b s}}+\left(M_{\eta_{b}}-E_{\eta_{b}}\right)=9.81 \mathrm{GeV}$ with error $= \pm 40 \mathrm{MeV}$. 
For $\Lambda_{b s s}\left(\frac{1}{2}^{+}\right)$we find $M_{\Lambda_{b s s}}=E_{\Lambda_{b s s}}+\frac{1}{2}\left(M_{\eta_{b}}-E_{\eta_{b}}\right)=5.45 \mathrm{GeV}$ with error $= \pm 30 \mathrm{MeV}$.

\section{Summary}

We presented our preliminary results of charmed and strange heavy mesons and baryons. For bottom quark we used NRQCD action corrected up to $O\left(v^{6}\right)$ in velocity and up to $O\left(a^{4}\right)$ in lattice spacing. For strange and charm quarks we used relativistic overlap and hisq actions. We simulated on $N_{f}=2+1+1$ HISQ gauge configurations generated by MILC collaboration. We found that mass we obtained for $B_{c}$ meson is in agreement with its experimental value. Also the mass of $\Omega_{b b b}$ baryon matches with the value reported by other groups. The strange $B$ meson mass deviates from its experimental value. However it is interesting to see splitting of $B_{s}$ and $B_{s}^{*}$.

\section{Acknowledgement}

Simulations have been carried out on the Proton cluster of School of Physical Sciences, NISER. We thank TIFR, Mumbai group for their overlap propagators. We also thank MILC collaboration for providing HISQ lattices. 


\section{References}

[1] G.P. Lepage, L. Magnea, C. Nakhleh, U. Magnea, K. Hornbostel, Phys. Rev. D46, 4052 (1992), hep-lat/9205007

[2] B.A. Thacker, G.P. Lepage, Phys. Rev. D43, 196 (1991)

[3] H.D. Trottier, Phys. Rev. D55, 6844 (1997), hep-lat/9611026

[4] C.T.H. Davies, K. Hornbostel, G.P. Lepage, A.J. Lidsey, J. Shigemitsu, J.H. Sloan, Phys. Rev. D52, 6519 (1995), hep-lat/9506026

[5] C.T.H. Davies, K. Hornbostel, A. Langnau, G.P. Lepage, A. Lidsey, J. Shigemitsu, J.H. Sloan, Phys. Rev. D50, 6963 (1994), hep-lat/9406017

[6] T. Burch (2015), 1502.00675

[7] S. Meinel, Phys. Rev. D82, 114514 (2010), 1008. 3154

[8] S. Meinel, W. Detmold, C.J.D. Lin, M. Wingate, PoS LAT2009, 105 (2009), 0909. 3837

[9] A. Bazavov et al. (MILC), Phys. Rev. D87, 054505 (2013), 1212 . 4768

[10] S. Basak, S. Datta, A.T. Lytle, M. Padmanath, P. Majumdar, N. Mathur, PoS LATTICE2013, 243 (2014), 1312.3050 